\title{
CIÊNCIANATURA
}

\section{Características da esteira turbulenta do modelo de um aerogerador}

Characteristics of the turbulent wake of a wind turbine model

${ }^{1}$ Adrián Roberto Wittwer, ${ }^{2}$ Rodrigo Dorado, ${ }^{2}$ Acir Loredo-Souza, ${ }^{2}$ Arthur Bones, ${ }^{2}$ Bruno Capeller, ${ }^{2}$ André Contini, ${ }^{2}$ Bardo Bodmann e ${ }^{2}$ Gervásio Degrazia

${ }^{1}$ Facultad de Ingeniería, Universidad Nacional del Nordeste, Resistencia, Argentina

${ }^{2}$ Universidade Federal de Santa Maria, Santa Maria, RS, Brasil

\section{Resumo}

Um estudo experimental das características da esteira turbulenta a sotavento do rotor de uma turbina eólica foi realizado no túnel de vento "Joaquim Blessmann" da UFRGS. O modelo da turbina foi desenvolvido na Universidade de Caxias do Sul e representa um aerogerador de três pás caracterizadas por um perfil aerodinâmico NACA 4412. Medições das flutuações de velocidade foram realizadas com um anemômetro de fio quente. A complexidade do escoamento turbulento é avaliada a partir dos perfis de velocidade média e flutuantes. A influência da turbulência do escoamento incidente e o processo de recomposição do escoamento são avaliados através das medições realizadas.

Palavras-chave: Turbinas eólicas. Túnel de vento. Turbulência

\section{Abstract}

An experimental study of the turbulent wake of a wind turbine model was realized at the "Joaquim Blessmann" wind tunnel of the UFRGS. The turbine model was developed at the Universidade de Caxias do Sul and it represents a three blade turbine characterized by a NACA 4412 aerodynamic profile. Measurements of the velocity fluctuations were realized by hot wire anemometry. Complexity of the turbulent flow is evaluated by mean and fluctuating velocity profiles. The influence of the incident flow turbulence and the flow reconstructing process are analyzed by the measurement results.

Keywords: Eolic turbines. Wind tunnel. Turbulence 


\section{Introdução}

A utilização da energia eólica está se difundindo devido aos múltiplos benefícios de suas aplicações. Entre as vantagens está a abundância desta fonte de energia, o baixo impacto ambiental e o baixo custo de manutenção dos equipamentos, de acordo com World Wind Energy Association (WWEA). Ao final de 2016 a capacidade de geração de energia eólica mundial instalada atingiu 486,6 GW, representando pouco mais de 4\% da capacidade total de geração no mundo, representatividade ainda pequena, porém vem aumentando gradativamente ao longo dos últimos anos.

A análise da interação fluido-estrutura entre o vento incidente e os aerogeradores requer a consideração de diferentes aspectos tais como as características do vento incidente, o déficit de velocidade provocado pelo próprio rotor e os níveis de turbulência na esteira. $\mathrm{O}$ escoamento turbulento nos parques eólicos caracteriza-se pela superposição de esteiras e as perdas do potencial eólico. Na literatura específica existem diferentes trabalhos indicando que uma turbina operando dentro de um parque pode sofrer perdas da potência gerada de até $40 \%$, quando comparada com a turbina operando individualmente (FRANDSEN et al., 2004). A complexidade de um fenômeno que inclui a coexistência de esteiras múltiplas, os efeitos da camada limite turbulenta, a topografia local e a estratificação térmica sugerem que o estudo do escoamento nos parques eólicos deveria utilizar todas as ferramentas disponíveis de forma complementária para sua melhor caracterização. A experimentação em túnel de vento com modelos reduzidos permite reproduzir os fenômenos característicos da interação sob condições controladas. Com relação a este tipo de estudos, existem trabalhos orientados a avaliar a estrutura da esteira dos aerogeradores (BARTL, 2011), e outros que analisam a turbulência efetiva de um parque eólico (CHAMORRO; PORTÉ-AGEL, 2011). Atualmente, são desenvolvidos modelo aerolásticos a escala para avaliar o comportamento dinâmico de rotores de aerogeradores (BAYATI et al., 2017).

Em um estudo experimental prévio (WITTWER et al., 2016) foram avaliadas as características espectrais da turbulência na esteira do modelo de um aerogerador mantendo a semelhança geométrica. Neste trabalho, as características do escoamento médio e da turbulência na esteira de um novo modelo considerando dois tipos de vento incidente são analisadas a partir de medições da componente longitudinal da velocidade flutuante com anemômetro de fio quente. Os testes foram desenvolvidos no túnel de vento "Joaquim Blessmann" da UFRGS e o modelo foi desenvolvido na Faculdade de Engenharia da Universidade de Caxias do Sul (UCS). A semelhança do modelo foi centrada principalmente nos aspectos aerodinâmicos relegando a similitude estritamente geométrica.

\section{Características do medelo da turbina}

Ao projetar asas de aeronaves, se considera que a asa tem uma distribuição de velocidade uniforme ao longo de sua extensão e o desempenho da asa é ótimo quando o ângulo de ataque está definido corretamente. Para otimizar o desempenho da turbina, o ângulo de passo $\beta$ deve ser determinado em função do raio $r$ da pá e nas posições mais afastadas do eixo de rotação, a componente de velocidade na direção do plano de rotação aumenta. A geometria ideal da pá pode ser projetada de acordo com a teoria de Schmitz, que permite gerar o comprimento de corda c e ângulo de passo ideais considerando parâmetros como velocidade do vento, quantidade de pás, raio da pá R, taxa de velocidade na ponta da pá TSR, ângulo de ataque ideal $\alpha$ e do coeficiente de sustentação CL, do perfil aerodinâmico escolhido (THUMTHAE; CHITSOMBOON, 2009).

A equação (1) é usada para calcular o ângulo de passo e a equação (2) é usada para calcular o comprimento de corda. Na figura 1, são indicadas as forças aerodinâmicas atuantes na turbina em operação. A potência máxima disponível pela energia cinética do vento P considerando um fluxo é dada pela equação (1), onde A é área do rotor e v1 a velocidade do vento antes de atingir a turbina.

$$
P=\frac{1}{2} \rho A v_{1}^{3}
$$

O vento atravessando o rotor da turbina é desacelerado devido a energia cinética do vento absorvida pelo gerador (figura 1). A potência mecânica PT da turbina é dada pela equação (2), onde v é a velocidade do vento que atravessa o rotor e v3 é a velocidade após ultrapassar o rotor.

$$
P_{T}=2 \rho a(1-a)^{2} v_{1}^{3} A
$$

As velocidades são:

$$
\begin{aligned}
& v=\frac{1}{2}\left(v_{1}+v_{3}\right) \\
& v_{3}=(1-2 a) v_{1}
\end{aligned}
$$

O fator de interferência axial a representa a perda de velocidade de vento à medida que se aproxima da pá. A eficiência de 
uma turbina eólica pode ser determinada pelo coeficiente de potência adimensional CP, definido pela equação (5).

$$
C_{P}=\frac{P_{T}}{P}
$$

O máximo coeficiente de potência teórico é 0,59 , conhecido como limite de Betz, atingido quando a $=1 / 3$. Devido a perdas do sistema como fricção dos rolamentos, vórtices de ponta de pá e perdas pelo centro do rotor, o coeficiente de potência real é sempre menor que o limite de Betz.
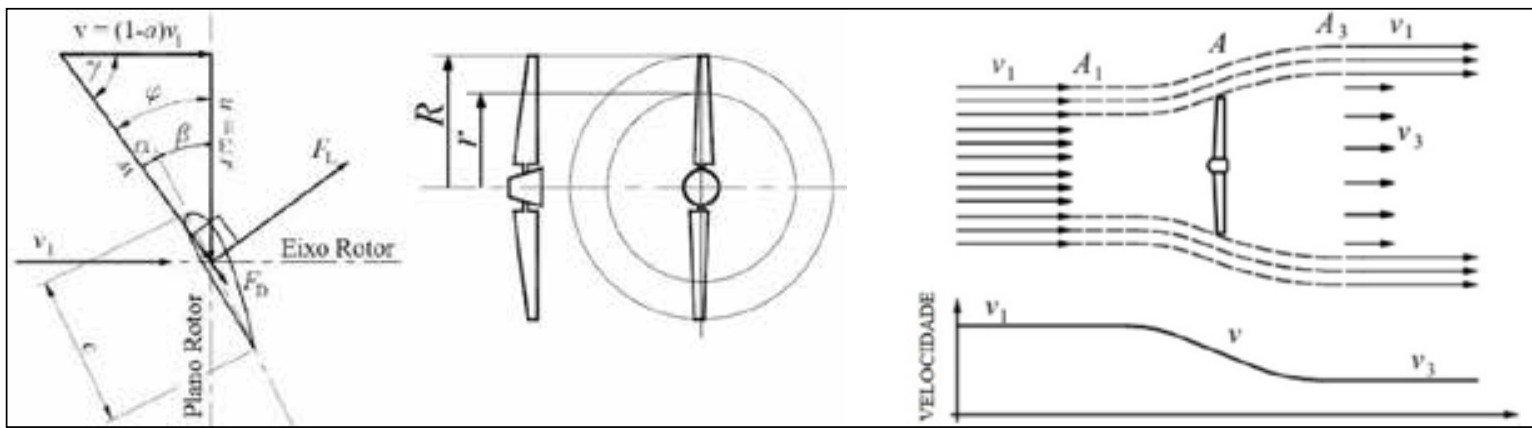

Figura 1 - Esquema de atuação de perfil aerodinâmico e velocidades em torno do rotor

O modelo foi projetado para representar um protótipo de turbina eólica de 3 palas, $100 \mathrm{~m}$ de altura da torre e $100 \mathrm{~m}$ de diâmetro de rotor utilizando uma escala de 1:150. As especificações do modelo são listadas na tabela 1 e foi adotado TSR de 5 em função das dimensões e das características do túnel de vento. A usinagem das pás foi feita no centro de usinagem para prototipagem CNC da UCS. A figura 2 mostra as principais dimensões e características do modelo. Na fotografia a direita pode-se observar um dispositivo de frenagem desenvolvido para aplicar um torque de valor conhecido ao eixo da turbina. $\mathrm{O}$ dispositivo possui um braço articulado que é tensionado contra o eixo pela ação de uma mola de tração.

Tabela 1 - Especificações da turbina

\begin{tabular}{c|c}
\hline Número de pás & 3 \\
\hline Diâmetro do rotor & $600 \mathrm{~mm}$ \\
\hline TSR & 5 \\
\hline Perfil aerodinâmico da pá & NACA 4412 \\
\hline
\end{tabular}
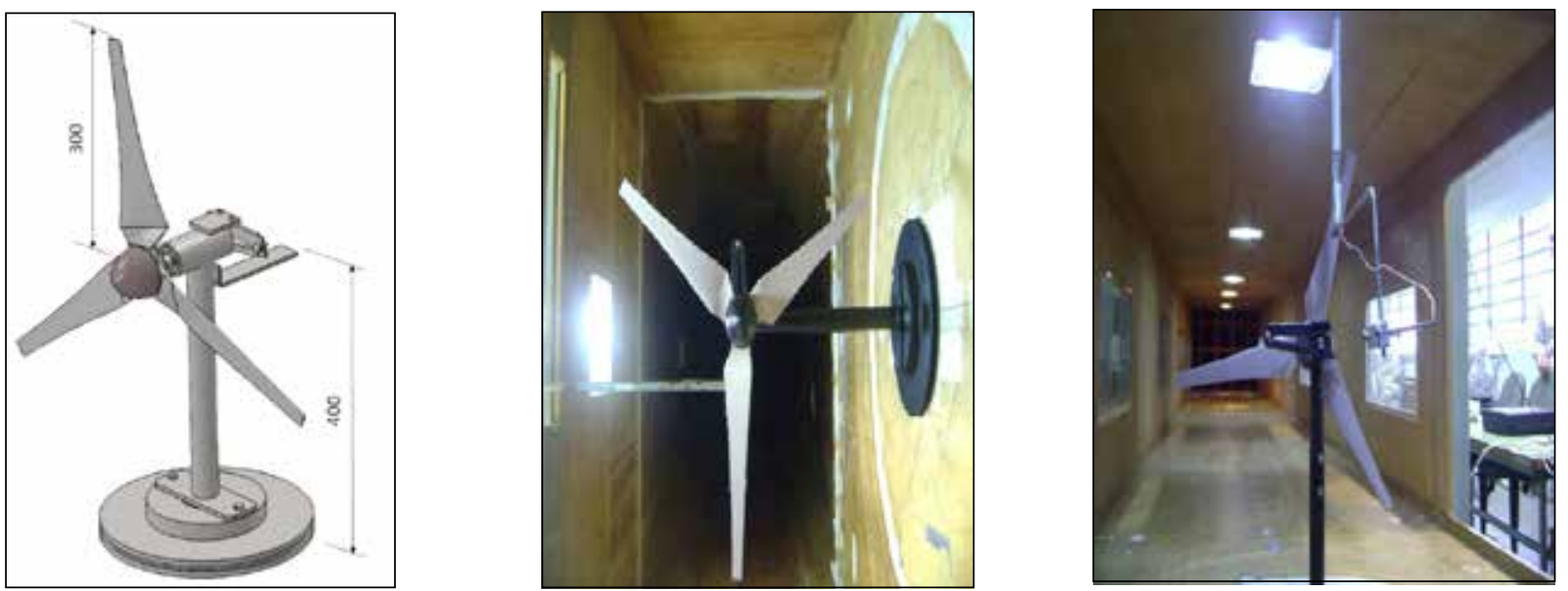

Figura 2 - Modelo do aerogerador.

\section{Descrição dos ensaios}

Os testes foram desenvolvidos no túnel de vento "Joaquim Blessmann" da UFRGS, do tipo retorno fechado. O túnel foi projetado especificamente para ensaios estáticos e dinâmicos de modelos de construções civis, permitindo a simulação física 
de ventos uniformes e ventos de camada limite. A câmara principal de ensaios possui secção transversal de dimensões iguais a 1,2 m $\times 0,8 \mathrm{~m}$. A velocidade máxima do escoamento de ar nesta câmara, com vento uniforme e suave, é de $42 \mathrm{~m} / \mathrm{s}$ (BLESSMANN, 1982).

As medições da velocidade flutuante foram realizadas com anemômetro de fio quente a distintas posições x a sotavento do rotor, obtendo-se perfis verticais e horizontais de velocidade média e do valor RMS das flutuações de velocidade. Às distâncias a sotavento do modelo variaram de $\mathrm{x}=160 \mathrm{~mm}$ até $5160 \mathrm{~mm}$. As series numéricas representativas da velocidade flutuante foram obtidas com uma frequência de amostragem de $2048 \mathrm{~Hz}$ durante 60 segundos. O valor do número de Reynolds definido com relação à velocidade média dos testes e o diâmetro do rotor é 2,25 × 105. A velocidade de rotação da turbina foi medida no início das medições, fixando de forma conveniente o tensor de frenagem mecânico em função do parâmetro adimensional TSR. El valor aproximado do TSR durante os ensaios foi 5 .

\section{Resultados}

Nesta seção são apresentados os diferentes resultados obtidos nos testes descritos. Primeiro são indicados os perfis de velocidade média nos diferentes pontos a sotavento do rotor e logo depois, são analisados os perfis da flutuação de velocidade. Em diferentes posições x são apresentados os perfis verticais e horizontais definidos em correspondência ao eixo do rotor.

\section{Velocidade média}

Na figura 3 são apresentados os perfis verticais de velocidade média com escoamento incidente uniforme turbulento, à esquerda, e escoamento uniforme suave, à direita. A coordenada vertical $\mathrm{z}=0$ corresponde aproximadamente à posição do eixo do rotor. Da mesma forma, na figura 4 são apresentados os perfis horizontais de velocidade média, onde a coordenada horizontal y $=0$ também corresponde ao eixo do rotor.
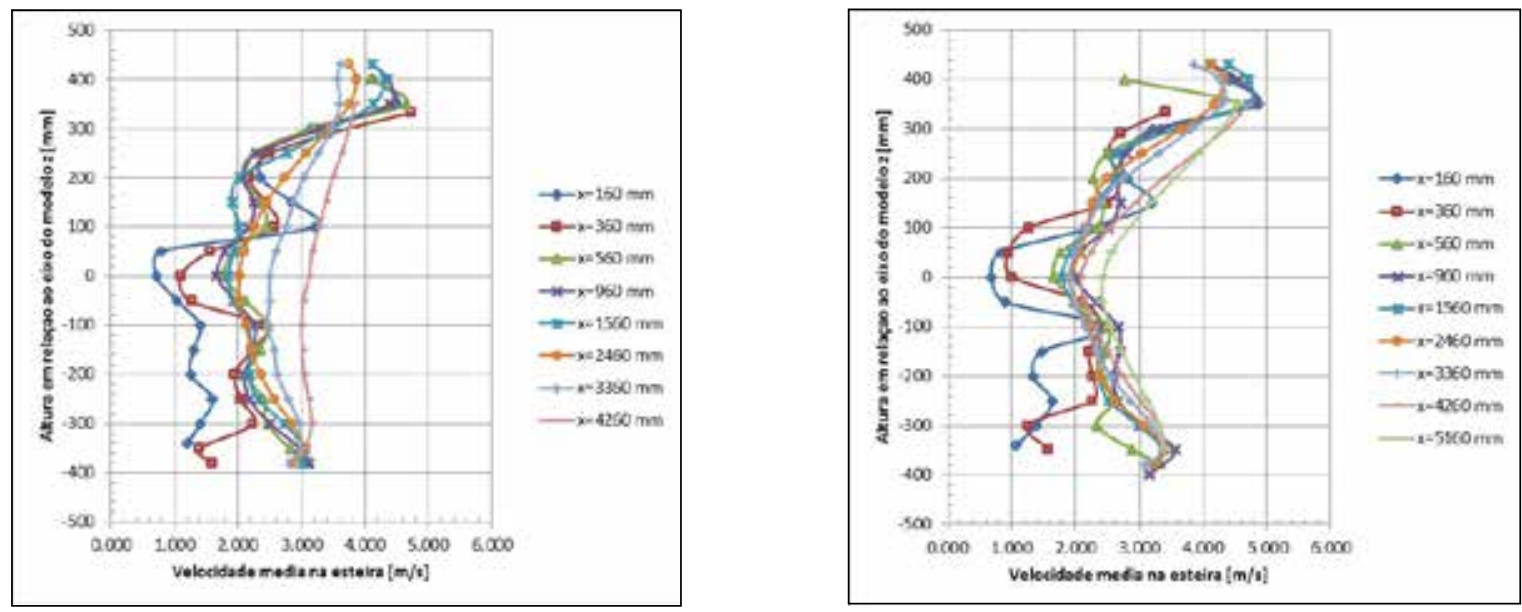

Figura 3 - Perfis verticais de velocidade média com escoamento incidente turbulento e suave.
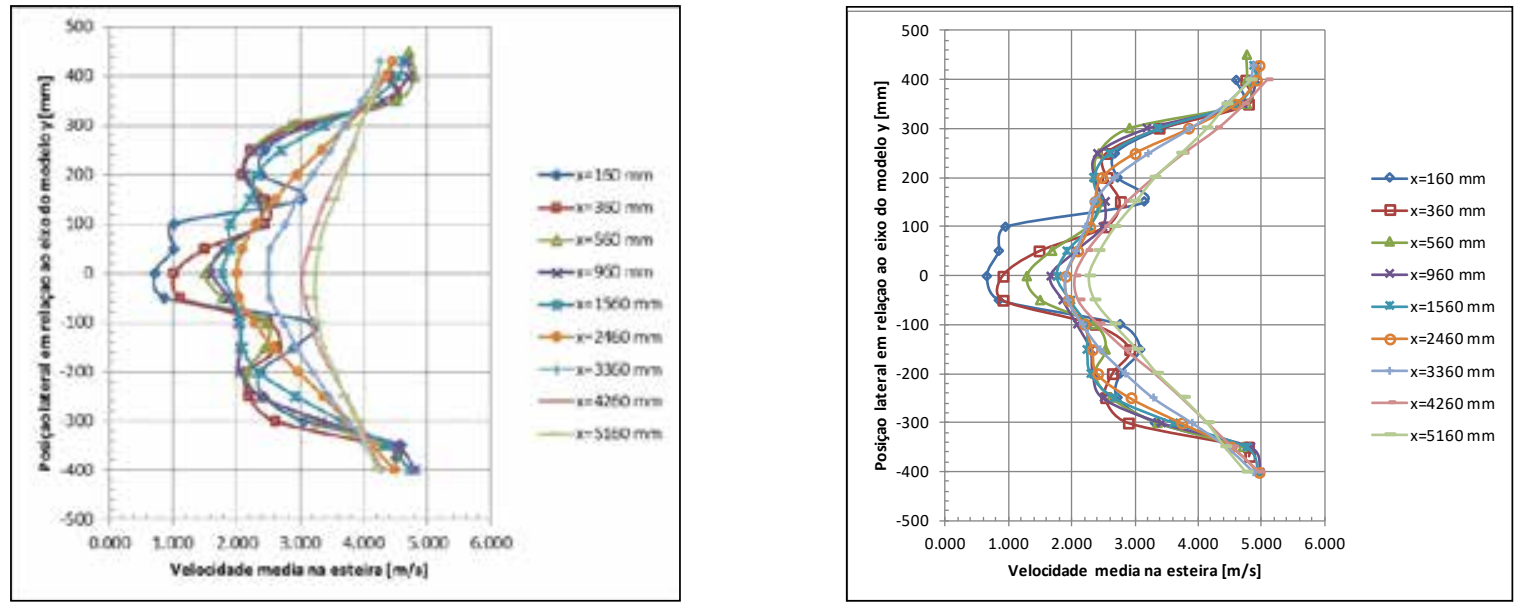

Figura 4 - Perfis horizontais de velocidade média com escoamento incidente turbulento e suave. 
Ainda considerando que o fio quente somente pode medir a componente longitudinal da velocidade, e possível observar a complexidade do escoamento nos pontos mais próximos do rotor. A falta de simetria nos perfis verticais claramente indica a presença da torre do modelo do gerador provocando maiores quedas de velocidade na parte inferior. Por outro lado, os perfis horizontais mostram boa simetria exceto nas proximidades do dispositivo de frenagem $(x=160 \mathrm{~mm}, \mathrm{z}= \pm 100 \mathrm{~mm})$. Nas posições mais afastadas do modelo é possível observar maior uniformidade do perfil no caso do vento turbulento. Isto indicaria que a recomposição do escoamento incidente acontece mais rapidamente quando a turbulência é maior.

\section{Flutuações de velocidade}

Os perfis verticais das flutuações de velocidade indicados na figura 5 foram obtidos com escoamento incidente uniforme turbulento (esquerda) e escoamento uniforme suave (direita), entanto, na figura 6 são apresentados os perfis horizontais. As coordenadas $\mathrm{y}=0 \mathrm{e} \mathrm{z}=0$ correspondem ao eixo do rotor.
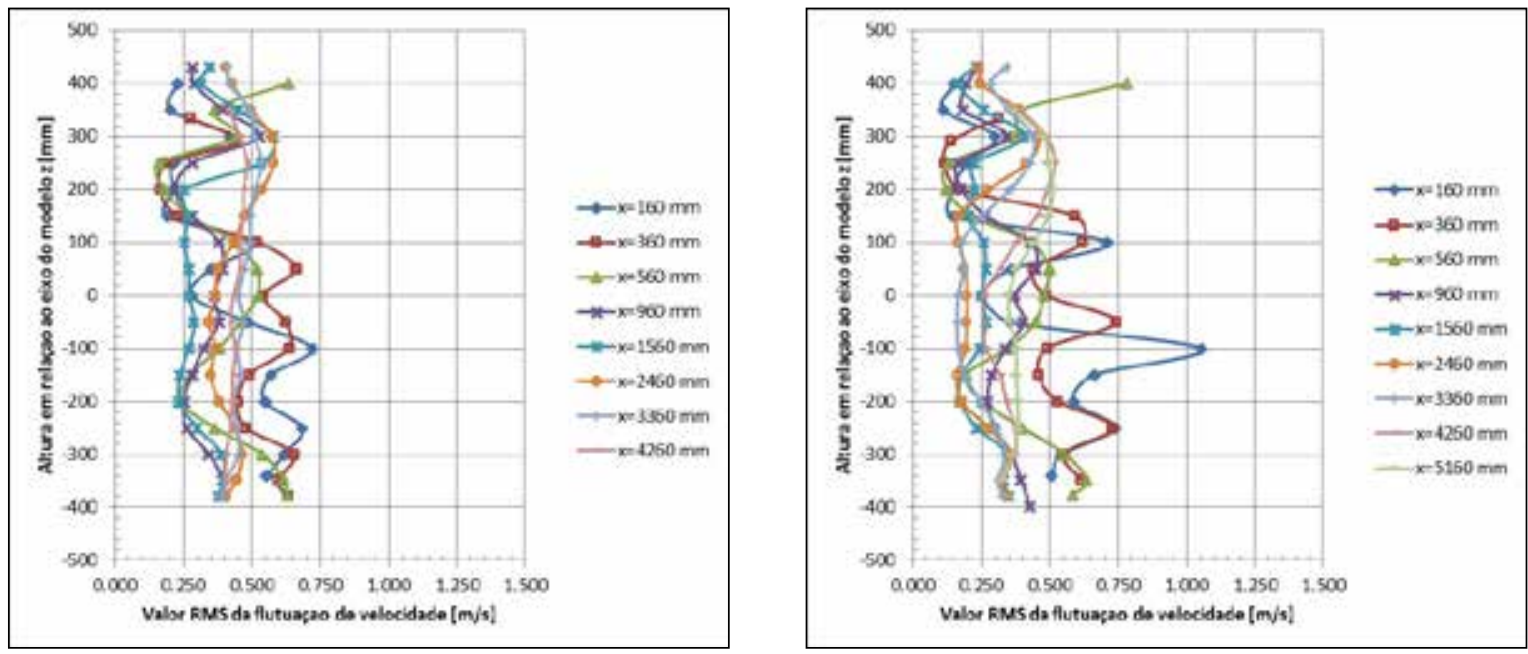

Figura 5 - Perfis verticais das flutuações de velocidade com escoamento incidente turbulento e suave.
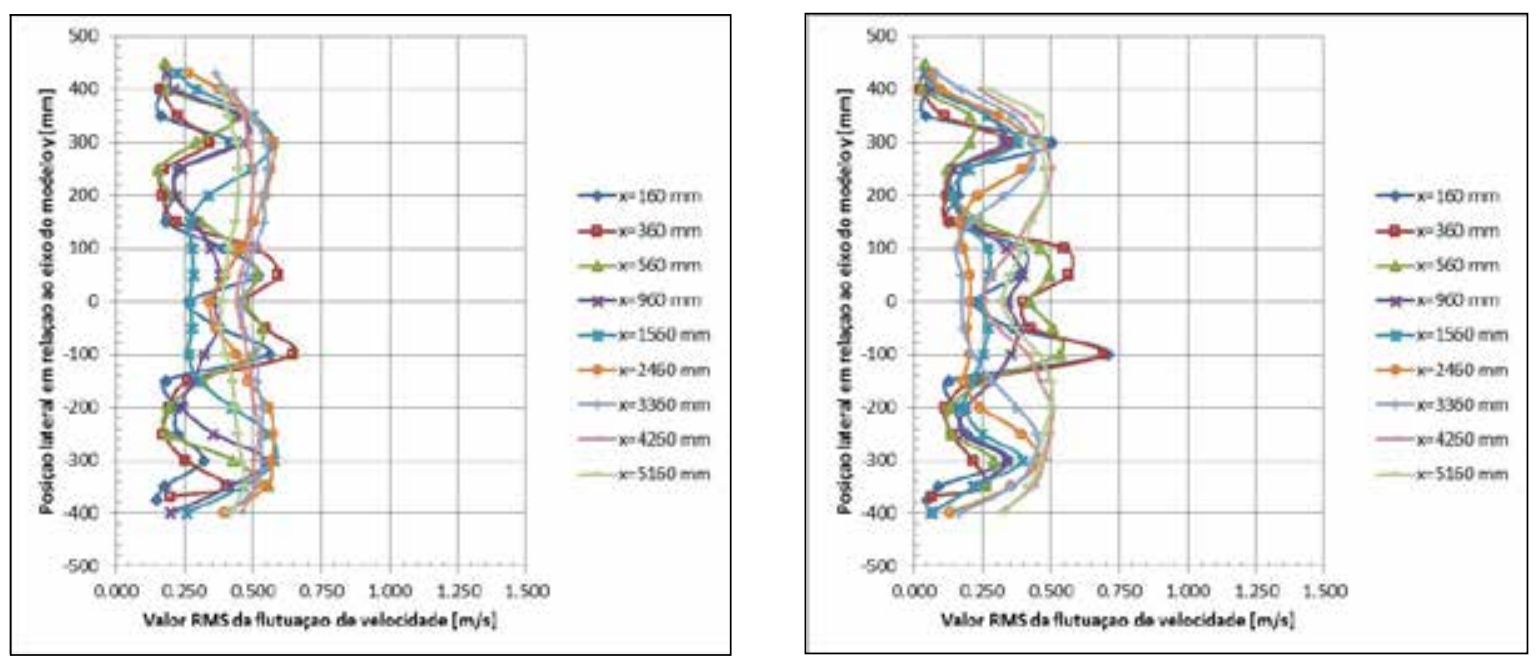

Figura 6 - Perfis horizontais das flutuações de velocidade com escoamento incidente turbulento e suave.

A complexidade do escoamento nos pontos mais próximos do rotor que foi mostrada na análise de velocidade média também pode ser observada nos desprendimentos de vórtices que indicam os perfis do valor RMS das flutuações de velocidade. Verifica-se também a falta de simetria nos perfis verticais das flutuações indicando a presença da coluna do modelo do gerador que provoca picos pelo desprendimento de vórtices que são maiores na parte inferior. Os perfis horizontais das flutuações também mostram boa simetria observando-se o desprendimento local de vórtices nas proximidades do dispositivo de frenagem. Nas posições mais afastadas do modelo, da mesma forma que no caso da velocidade média, é possível observar maior uniformidade do perfil do valor RMS das flutuações no caso do vento turbulento. A observação mais interessante é que não se evidencia uma recomposição dos níveis de turbulência. 


\section{Conclusões}

O projeto de turbinas de aerogeradores é realizado com base em considerações aerodinâmicas que simplificam a configuração do escoamento ultrapassando o rotor. Estudos experimentais permitem observar a complexidade das esteiras turbulentas geradas a sotavento do rotor. Em particular, este estudo permite identificar zonas de maior complexidade a partir da avaliação do escoamento médio e dos níveis de turbulência na esteira do modelo a escala reduzida de um aerogerador. A influência da turbulência do escoamento incidente é também analisada a partir dos resultados experimentais. Finalmente, pode-se verificar que a recomposição do escoamento médio não necessariamente implica que os níveis de turbulência atingiram os valores iniciais.

\section{Agradecimentos}

Os autores agradecem ao CNPq pelo suporte financeiro para o desenvolvimento deste trabalho.

\section{Referências}

BARTL J. Wake Measurements behind an array of two Model Wind Turbines [thesis], KTH School of Industrial Engineering and Management Energy Technology, EGI-2011-127 MSC EKV 866, Division of Heat and Power Technology, SE-100 44 Stockholm; 2011.

BAYATI I, BELLOLI M, BERNINI L, ZASSO A. Aerodynamic design methodology for wind tunnel tests of wind turbine rotors. J. Wind Eng. Ind. Aerodyn. 2017; 167: 217-227.

BLESSMANN J. The Boundary Layer Wind Tunnel of UFRGS. J. Wind Eng. Ind. Aerodyn. 1982; 10: $231-248$.

CHAMORRO L, PORTÉ-AGEL F. Turbulent Flow Inside and Above a Wind Farm: A Wind-Tunnel Study. Energies. 2011; 4: 1916-1936.

FRANDSEN S, BARTHELMIE R, PRYOR S, RATHMANN O, LARSEN S, HØJSTRUP J et al. Analytical modeling of wind speed deficit in large offshore wind farms. In: Proceedings of EWEC; 2004 Nov 22-25, London, UK.

THUMTHAE C, CHITSOMBOON T. Optimal angle of attack for untwisted blade Wind turbine. Renewable Energy. 2009; 34: 1279-1284.

WITTWER A, DORADO R, ALVAREZ Y ALVAREZ G, DEGRAZIA G, LOREDO-SOUZA A, BODMANN B. Flow in the Wake of Wind Turbines: Turbulence Spectral Analysis by Wind Tunnel Tests. American Journal of Environmental Engineering. 2016; 6: 109-115.

\section{Adrián Roberto Wittwer}

Facultad de Ingeniería, Universidad Nacional del Nordeste, Resistencia, Argentina E-mail: a_wittwer@yahoo.es

Rodrigo Dorado

Universidade Federal de Santa Maria, Santa Maria, RS, Brasil E-mail: dorado@ste-pdi.com.br

Acir Loredo-Souza

Universidade Federal de Santa Maria, Santa Maria, RS, Brasil E-mail: acir@ufrgs.br

Arthur Bones

Universidade Federal de Santa Maria, Santa Maria, RS, Brasil E-mail: arthurbones_43@hotmail.com 


\section{Bruno Capeller}

Universidade Federal de Santa Maria, Santa Maria, RS, Brasil E-mail: brunocapeller@gmail.com

André Contini

Universidade Federal de Santa Maria, Santa Maria, RS, Brasil E-mail: accontini@ucs.br

\section{Bardo Bodmann}

Universidade Federal de Santa Maria, Santa Maria, RS, Brasil E-mail: bardo.bodmann@ufrgs.br

\section{Gervásio Degrazia}

Universidade Federal de Santa Maria, Santa Maria, RS, Brasil

E-mail: gervasiodegrazia@gmail.com 